\title{
Intraspecific and Interspecific Interactions Mediated by a Phytotoxin, (-)-Catechin, Secreted by the Roots of Centaurea maculosa (Spotted Knapweed)
}

\author{
Tiffany L. Weir • Harsh Pal Bais • Jorge M. Vivanco
}

Published online: 16 July 2009

(C) Springer Science + Business Media, LLC 2009

Erratum to: J Chem Ecol (2003) 29:2397-2412

DOI 10.1023/A:1026313031091

This article has been retracted by Tiffany L. Weir and Jorge M. Vivanco.

Reason: After a reevaluation of this work, we no longer have confidence in several key observations and therefore, retract this paper. Specifically, the reported finding in Perry et al. ( $\mathrm{J}$ of Chemical Ecology 33:2337$2344,2007)$ of catechin in experimental blanks, suggest an exogenous source of catechin in our laboratory. The absence of experimental blanks in the current study

along with the unreproducibly high levels of catechin reported from in vitro grown C. maculosa plants have led us to believe that the reported catechin may have originated from non-plant sources. In addition, a finding that some of our commercially purchased catechin was contaminated with 2,4-D along with our observation that catechin caused callus formation in some seedlings suggests that at least some of these experiments may have been conducted using a contaminated source. Because we cannot be sure that contaminated catechin was not used, the results of the phytotoxicity and germination inhibition experiments are meaningless.

The online version of the original article can be found at http://dx.doi. org/10.1023/A:1026313031091.

T. L. Weir $\cdot$ H. P. Bais $\cdot$ J. M. Vivanco

Department of Horticulture and Landscape Architecture,

Colorado State University,

Fort Collins, Colorado 80523-1173, USA

J. M. Vivanco $(\bowtie)$

Graduate Program in Ecology, Colorado State University,

Fort Collins, Colorado 80523-1173, USA

e-mail: jvivanco@lamar.colostate.edu 